\title{
High Resolution X-Ray Spectra for Chemical Speciation in the SEM
}

Katherine Schreiber ${ }^{1}$, Daniel McNeel$^{2}$, Katrina Koehler ${ }^{2}$, Chandler Smith ${ }^{2}$, Benjamin Stein ${ }^{2}$, Gregory Wagner ${ }^{2}$, Eric Bowes ${ }^{2}$, Lei Xu ${ }^{2}$, Christopher Fontes ${ }^{2}$, Enrique Batista ${ }^{2}$, Ping Yang ${ }^{2}$, Michael Rabin ${ }^{2}$, Mark Croce ${ }^{2}$ and Matthew Carpenter ${ }^{2}$

${ }^{1}$ Los Alamos National Laboratory, Los Alamos, New Mexico, United States, ${ }^{2}$ Los Alamos National Laboratory, United States

To discern the oxidation state and chemical speciation of a material with nanoscale spatial resolution, $\mathrm{x}$-ray spectra obtained with wavelength-dispersive techniques at synchrotrons have so far been necessary [1-3]. Energy dispersive x-ray detectors mounted in scanning electron microscopes (SEMs) are by comparison commonplace, time-efficient, and easy to use, but have an energy resolution in the $100 \mathrm{eV}$ range, too broad for chemical speciation [2,3]. Here, we report recent progress on a new instrument which will combine high energy resolution capabilities (on the order of a few eV FWHM) with high efficiency (the ability to acquire a spectrum at full energy bandwidth on a short time scale) and the high spatial resolution (nanometer scale) of a scanning electron microscope [4]. This instrument is referred to as Hyperspectral X-ray Imaging (HXI). The HXI instrument will greatly enhance nondestructive chemical analysis of tiny samples, of particular benefit to the field of nuclear safeguards. Other areas of study which require precise knowledge of oxidation state and chemical speciation, such as the analysis of geological samples and novel nanomaterials, can benefit from HXI as a time and resource efficient alternative to synchrotron capabilities.

The primary feature of the detector is an array of transition edge sensor (TES) microcalorimeters, which operate at milliKelvin temperature and obtain extremely high resolution electromagnetic spectra [5,6]. Currently we operate an x-ray detector for the measurement of element oxidation state and chemical speciation in bulk compounds, called X1 [7]. A commercial x-ray generator illuminates bulk samples within the sample chamber, allowing us to obtain spectra up to about 20 $\mathrm{keV}$. Figure 1 shows a representative spectrum of lead metal. In this spectrum, several $\mathrm{Pb}$ lines are visible, in addition to the prominent $\mathrm{Al} \mathrm{K}_{\alpha}$ line arising from the sample holder. The peak corresponding to the $\mathrm{Si} \mathrm{K}_{\alpha}$ line at $1.74 \mathrm{keV}$ has a FWHM of $5.45 \mathrm{eV}$. The narrow FWHM of these peaks in principle lets us detect tiny shifts on the order of $\mathrm{eV}$ due to elements in different oxidation states [1]. From this we can extrapolate information about the chemical compounds of which an element may be a part. With X1, we are constructing a spectral library of energy shifts arising from the electron configuration of atoms in different chemical compounds.

The HXI will take the x-ray microcalorimeter array a step further, integrating a detector with a TES array into an SEM. This detector array, referred to as a microsnout, has been designed and is ready for fabrication. This snout will be inserted into a JEOL JSM-7200F scanning electron microscope. In this SEM, we can obtain accelerating voltage up to $30 \mathrm{kV}$ and a beam current of $200 \mathrm{nA}$, with spatial resolution down to approximately $1 \mathrm{~nm}$. The TES microsnout will be cooled by a BlueFors SD dilution refrigerator, capable of maintaining milliKelvin temperatures on a continuous basis. A 
diagram of the detector assembly and mounted dilution refrigerator at the SEM is shown in Figure 2. The tip of the microsnout is tapered and will be mounted for insertion and retraction from the device. When fully inserted, the detector array will be approximately $55 \mathrm{~mm}$ from the sample. The detector mounted on the dilution refrigerator will be mechanically decoupled from the SEM for best vibration isolation, with a protective latch to avoid damage to either instrument due to movement of the sample stage relative to the detector. With a typical beam current, we will be able to obtain up to 200 counts per second per pixel. This will allow for efficient data acquisition in the desired energy regimes. The snout acquires a full energy spectrum at one time, allowing a more efficient measurement than a similar resolution wavelength dispersive spectrometer. This ability permits the acquisition of high resolution data on a timescale of hours. The cryostat and SEM are procured, and the designs for the snout and associated shielding are in a mature phase. Assembly and initial testing are expected to be completed by late 2021 .

The microsnout will have three sets of pixels with $\mathrm{Au}$ absorbers and one set of Bi absorbers optimized for three energy ranges. One set will be optimized with small TES absorbers sensitive to $\mathrm{x}$-rays of 0.15 to $2 \mathrm{keV}$, two sets of medium energy pixels will be sensitive to $\mathrm{x}$-rays of energies of 0.2 to $5 \mathrm{keV}$, and one set of large pixels with $\mathrm{Bi}$ absorbers for x-rays of 0.5 to $18 \mathrm{keV}$. This will span a sizable portion of the $\mathrm{x}$-ray spectrum and gain signatures of many elements of interest. As in $\mathrm{X} 1$, we will be able to measure the $\mathrm{K}$ lines of many light elements, and the $\mathrm{M}$ and $\mathrm{L}$ lines of many heavy elements, with peak FWHMs on the order of eV.

A major focus of ongoing work will be to seamlessly integrate the data acquisition with a nearly simultaneous co-adding of spectra, energy calibration, and spatial mapping of the spectrum associated with each pixel obtained by the SEM. A machine learning effort towards an accurate and efficient energy calibration is a near-term goal of the project. We plan to implement a machine learning engine utilizing pattern recognition for key spectral features. The engine will identify major lines based on spacing and absorption edges for energy calibration in real time. This will permit an efficient and automated map of chemical composition for every pixel in the acquired sample image.

LA-UR-21-21438 


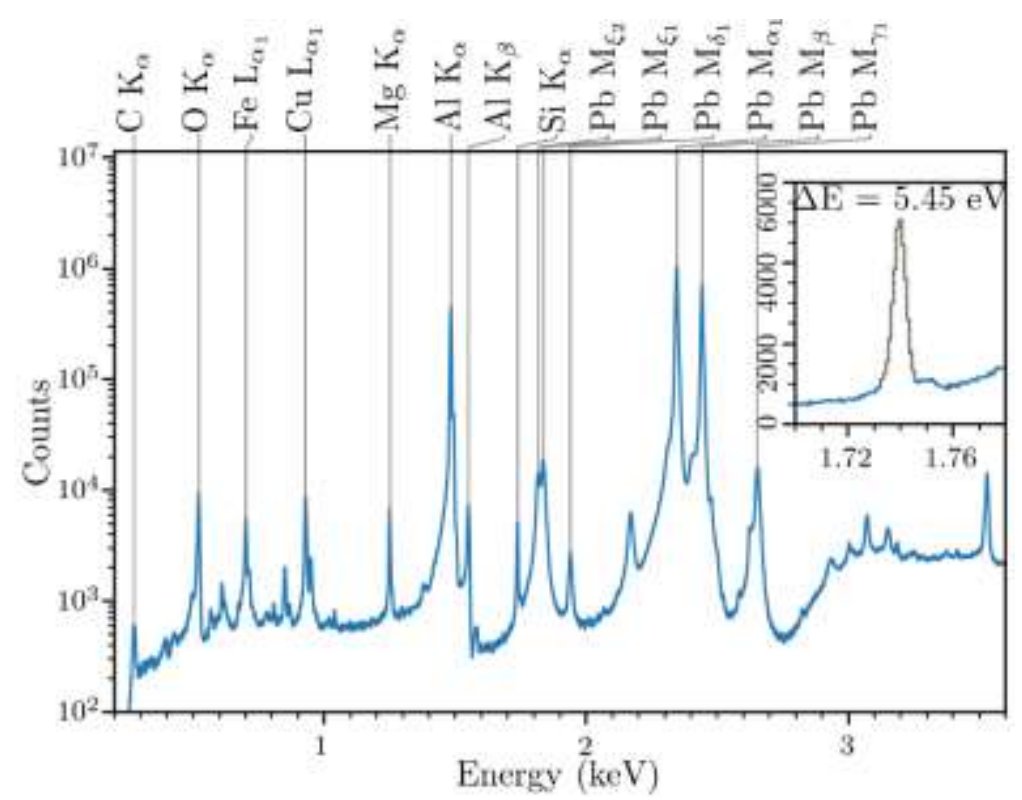

Figure 1. Sample spectrum of a $\mathrm{Pb}$ sample measured using $\mathrm{X} 1$. Prominent lines here are labeled. Inset: The $\mathrm{Si} \mathrm{K}_{\boldsymbol{\alpha}}$ line at $1.74 \mathrm{keV}$ displays a representative narrow peak, with a FWHM of $5.45 \mathrm{eV}$.
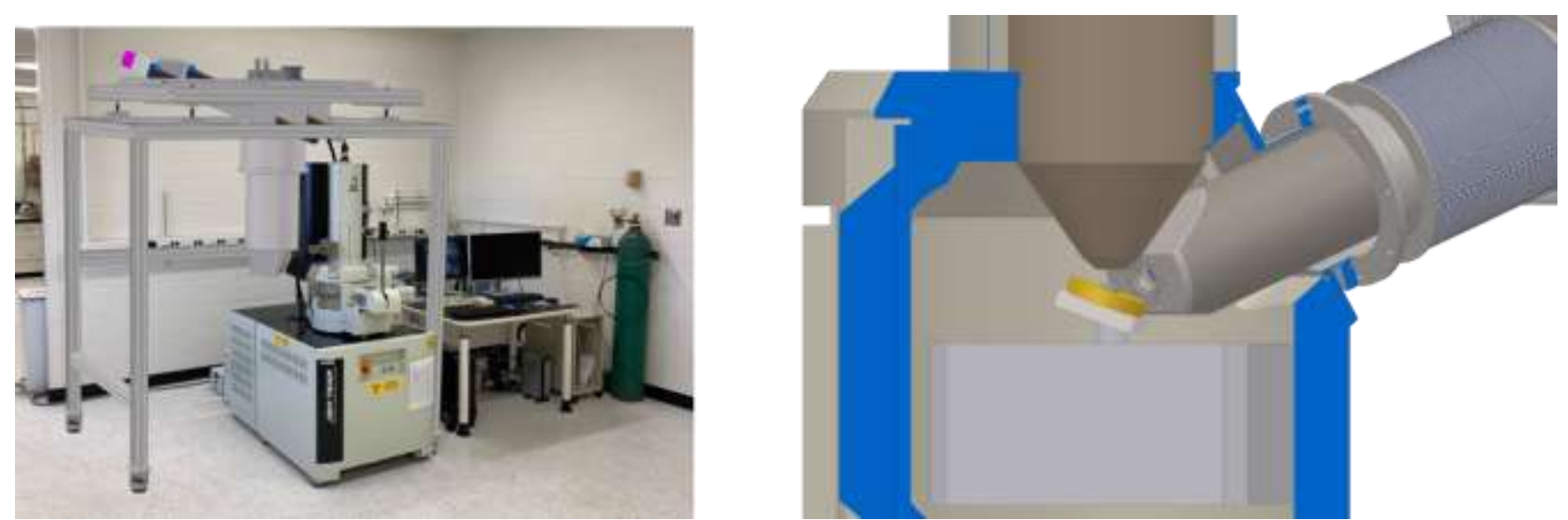

Figure 2. Left: A rendering of the dilution refrigerator with microsnout assembly coupled to the scanning electron microscope. The dilution refrigerator is the white cylinder mounted on a sliding track in the frame in the rendering. The detector microsnout protrudes from the refrigerator bottom, and in this picture tt is inserted into the SEM. Right: A close-up rendering of the detector snout inside the SEM chamber, with an effective distance of $50 \mathrm{~mm}$ from the beam spot to the detector array chip. The sample pin stub on the yellow sample stage is shown at a working distance of $10 \mathrm{~mm}$ and tilt of $20^{\circ}$.

References

[1] Y. V. Lomachuk and A. V. Titov. Phys. Rev. A 88, 062511 (2013).

DOI:10.1103/PhysRevA.88.062511

[2] P. Kuisma-Kursula, X-Ray Spectrom. 29, 111-118 (2000), DOI:10.1002/(SICI)1097-

4539(200001/02)29:1<111::AID-XRS408 >3.0.CO;2-W

[3]D.E. Newbury and N.W.M. Ritchie. J. Mater. Sci. 50, 493 (2015). DOI: 10.1007/s10853-014-8685-2 
[4] M. Carpenter, M. Croce, C. Smith, and K. Koehler. Microsc. Microanal. 26 (Suppl 2) (2020). DOI:10.1017/S143192762001369

[5] J.N. Ullom and D.A. Bennett. Supercond. Sci. Technol. 28, 084003 (2015). DOI::10.1088/09532048/28/8/084003

[6] D.A. Wollman, K.D. Irwin, G.C. Hilton, L.L. Dulcie, D.E., Newbury, and J.M. Martinis. J.

Microscopy. 188, 196 (1997). DOI: 10.1046/j.1365-2818.1997.2670824.x

[7] M.H. Carpenter, M.P. Croce, Z.K. Baker, E. R. Batista, M. P. Caffrey, C. J. Fontes, K. E. Koehler, S. E. Kossmann, K. G. McIntosh, M. W. Rabin, B. W. Renck, G. L. Wagner, M. P. Wilkerson, P. Yang, M. D. Yoho, J. N. Ullom, D. A. Bennett, G. C. O'Neil, C. D. Reintsema, D. R. Schmidt, G. C. Hilton, D. S. Swetz, D. T. Becker, J. D. Gard, J. Imrek, J. A. B. Mates, K. M. Morgan, D. Yan, A. L. Wessels, R. H. Cantor, J. A. Hall, and D.T. Carver. J . Low Temp. Phys. 200, 437 (2020). DOI: 10.1007/s10909-02002456-9 